\title{
Caracterización del hábitat de Amoreuxia wrightii (Bixaceae), una especie en peligro de extinción en el noreste de México
}

\section{Habitat characterization of Amoreuxia wrightii (Bixaceae), a species of Northeastern Mexico at risk}

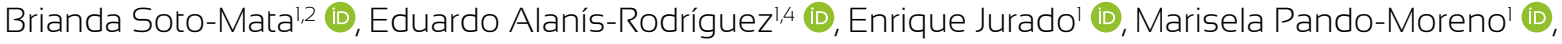 \\ Víctor Molina-Guerra² (D), Alejandro Alcalá-Rojas³ (D), Jonathan J. Marroquín-Castillo' (i)
}

1 Universidad Autónoma de Nuevo León, Facultad de Ciencias Forestales, Carretera Linares-Cd. Victoria km 145, Apdo. postal 41, 67700 Linares, Nuevo León, México.

2 RENAC, S.A. de C.V., Linares, Nuevo León, México.

3 Complejo Siderúrgico TERNIUM, Pesquería, Nuevo León, México.

4 Autor para la correspondencia: eduardo.alanisrd@uanl.edu.mx

Citar como:

Soto-Mata, B., E. Alanís-Rodríguez, E. Jurado, M. Pando-Moreno, V. Molina-Guerra, A. Alcalá-Rojas y J. J. Marroquín-Castillo. 2018. Caracterización del hábitat de Amoreuxia wrightii (Bixaceae), una especie en peligro de extinción en el noreste de México. Acta Botanica Mexicana 122: 21-31. DOl: http://dx.doi.org/10.21829/ abm122.2018.1196

Recibido: 11 de diciembre de 2016 Revisado: 22 de mayo de 2017. Aceptado: 23 de agosto de 2017. Primero en línea: 4 de octubre de 2017 Publicado: 1 de enero de 2018.

DOI

http://dx.doi.org/10.21829/abm122.2018.1196

\section{Resumen}

Antecedentes y Objetivos: En México, Amoreuxia wrightii se encuentra en estatus de peligro de extinción de acuerdo a la NOM-059-SEMARNAT-2010. En este estudio se realizó la caracterización de poblaciones naturales de $A$. wrightii, mediante un análisis de la flora asociada y las propiedades físicas y químicas de los suelos donde se desarrollan en dos comunidades contrastadas desde el punto de vista de la cobertura del dosel en el matorral tamaulipeco en el noreste de México.

Métodos: En 2014 se establecieron 10 sitios de muestreo en dos ambientes diferenciados por la estructura de la vegetación: cinco sitios en asociaciones vegetales con cobertura vegetal densa y cinco sitios en áreas perturbadas con suelo desnudo. En cada área se evaluó la estructura vegetal y variables edáficas y se realizó un Análisis de Correspondencia Canónica.

Resultados clave: De acuerdo a la relación con otras especies, A. wrightii se asoció con especies pioneras, que emergen después de un disturbio, con el dominio de pastos como Aristida purpurea, Cenchrus ciliaris, Cynodon dactylon, Panicum obtusum, especies de herbáceas Gutierrezia sarothrae, Gymnosperma glutinosum y Parthenium hysterophorus, así como arbustos de porte bajo como Acacia amentacea, $A$. berlandieri y Eysenhardtia texana. De acuerdo con las variables edáficas, se observó que A. wrightii prefiere suelos arcillosos y limosos, con suelos con $\mathrm{pH}$ ligeramente alcalinos y con densidad aparente alta. Ningún individuo de $A$. wrightii fue registrado en sitios que presentaron suelos con altos contenidos de arena y altos contenidos de materia orgánica.

Conclusiones: Amoreuxia wrightii fue más abundante en áreas con nula cobertura, desprovistas de vegetación, sobre suelos con bajo contenido de materia orgánica y altos contenidos de $\mathrm{pH}$, limo, arcilla y densidad aparente. De acuerdo a la relación con otras especies, se asoció con especies pioneras, que emergen después de un disturbio.

Palabras clave: análisis de correspondencia canónica, áreas con disturbio, cobertura aérea, Nuevo León.

\section{ABSTRACT:}

Background and Aims: In Mexico, Amoreuxia wrightii is endangered according to NOM-059-SEMARNAT-2010. In this study the characterization of natural populations of $A$. wrightii, which grow in two contrasting communities taking into account the canopy cover in the Tamaulipan thornscrub in northeastern Mexico, was performed by analysis of associated flora and physical and chemical properties of soils.

Methods: In 2014, 10 sampling sites were established in two environments differentiated by vegetation structure: five sites in plant associations with dense vegetation cover and five sites in disturbed areas with bare soil. In each area the vegetation structure and edaphic variables were evaluated and a Canonical Correspondence Analysis was performed.

Key results: According to the relationship with other species, $A$. wrightii was associated with pioneer species that emerge after disturbance, dominated by grasses like Aristida purpurea, Cenchrus ciliaris, Cynodon dactylon, Panicum obtusum, herbs such as Gutierrezia sarothrae, Gymnosperma glutinosum and Parthenium hysterophorus, and low shrubs like Acacia amentacea, A. berlandieri and Eysenhardtia texana. According to edaphic variables, it was observed that $A$. wrightii preferred clayey and silty soils, soils with slightly alkaline $\mathrm{pH}$ and high bulk density. No individual of $A$. wrightii was recorded at sites that had soils with high sand content and high content of organic matter.

Conclusions: Amoreuxia wrightii was more abundant in areas with no coverage, devoid of vegetation, soils low in organic matter and high in $\mathrm{pH}$, silt, clay and bulk density. According to the relationship with other species, it was associated with pioneer species, which emerge after a disturbance.

Key words: canonical correspondence analysis, disturbed sites, plant cover, Nuevo León. 


\section{INTRODUCCIÓN}

La familia Bixaceae agrupa plantas perennes, comprende 25 especies en cuatro géneros: Amoreuxia DC., Bixa L., Cochlospermum Kunth y Diegodendron Capuron. El género Amoreuxia se compone de cuatro especies: A. gonzalezii Sprague \& L. Riley, A. malvifolia A. Gray, A. palmatifida DC. y A. wrightii A. Gray, todas ellas distribuidas en México, pero no limitadas al país (Poppendieck, 1980; 1981; Pío-León et al., 2014).

En Nuevo León ocurren dos especies, A. palmatifida y $A$. wrightii, cuya distribución se restringe a la Planicie Costera del Golfo y sus límites con la Sierra Madre Oriental (Velazco, 2009). Se tienen registros de ejemplares (Herbario de la Facultad de Ciencias Forestales, UANL) colectados en Linares; además, Velazco (2009) registró $A$. palmatifida y A. wrightii en los municipios Bustamante, Lampazos de Naranjo y Anáhuac, Nuevo Léon.

Amoreuxia wrightii es una especie herbácea que se desarrolla principalmente en las regiones semiáridas (Cedano Maldonado, 2000; Velazco, 2009) y suele crecer en sitios perturbados (Calderón de Rzedowski, 1994). Se distribuye desde Texas, Estados Unidos de América, hasta el estado de Veracruz en áreas con suelos yesosos con altos contenidos de caliche (Henrickson y Johnston, 1997), así como en suelos arcillosos, planicies con suelos limosos (Correll y Johnston, 1970) y aún en pantanos (Sousa Pereira et al., 2013).

En México, A. wrightii se encuentra en estatus de peligro de extinción de acuerdo a la NOM-059-SEMARNAT-2010 (SEMARNAT, 2010). En esta categoría se encuentran las especies cuyas áreas de distribución o tamaño de sus poblaciones en el territorio nacional han disminuido drásticamente poniendo en riesgo su viabilidad biológica en todo su hábitat natural (SEMARNAT, 2010).

Debido al escaso conocimiento que existe sobre el hábitat donde prosperan las poblaciones de Amoreuxia wrightii, y que es una especie con estatus dentro de la NOM-059-SEMARNAT-2010 (SEMARNAT, 2010), los objetivos de este trabajo fueron caracterizar sus poblaciones naturales determinando las propiedades físicas y químicas de los suelos donde se desarrollan, así como la diversidad de la flora asociada en dos comunidades contrastadas desde el punto de vista de la cobertura del dosel en el matorral tamaulipeco en el noreste de México.

\section{Materiales y Métodos}

\section{Descripción del área de estudio}

El estudio se desarrolló en el Área de Conservación de Flora y Fauna Silvestre del Complejo Siderúrgico Ternium, en el municipio Pesquería, Nuevo León (Noreste de México), entre las coordenadas $25^{\circ} 44^{\prime} 32.17 " \mathrm{~N}$, y 99 57'45.23"W, con una altitud de 304 m (Fig. 1).

El clima corresponde al tipo $\mathrm{Cx}^{\prime}$ semicálido subhúmedo con lluvias escasas todo el año, con una precipitación media anual fluctuante entre 600 y $800 \mathrm{~mm}$ y una temperatura promedio anual de $22^{\circ} \mathrm{C}$. La temperatura media más alta se registra en el mes de julio con $30^{\circ} \mathrm{C}$; y la mínima en los meses de enero y diciembre con 14 y 15 ${ }^{\circ} \mathrm{C}$ (INEGI, 2014).

Los tipos de suelos son de origen aluvial, profundos, muy arcillosos, con contenido que oscilan de 40 a $65 \%$ en todo el perfil. En la época de sequía presentan grietas anchas y profundas que alcanzan, en ocasiones, más de $6 \mathrm{~cm}$ de ancho y $100 \mathrm{~cm}$ de profundidad. Destacan los Xerosoles, Feozems, y los Vertisoles (INEGI, 1986). La vegetación dominante pertenece al matorral espinoso tamaulipeco, el cual está dominado por especies como Acacia amentacea DC., Leucophyllum frutescens (Berland.) I.M. Johnst., Cordia boissieri A. DC. y Vachellia farnesiana (L.) Wight \& Arn. (Estrada et al., 2004; Alanís et al., 2013).

\section{Evaluación de la vegetación}

En el verano del año 2014 se establecieron 10 sitios de muestreo en dos ambientes diferenciados por la estructura de la vegetación: cinco sitios en asociaciones vegetales con cobertura vegetal densa y cinco en áreas perturbadas con suelo desnudo. La asociación con cobertura densa presenta árboles y arbustos maduros con una cobertura de copa de $9345 \mathrm{~m}^{2} / \mathrm{ha}$. El área perturbada tiene un historial 


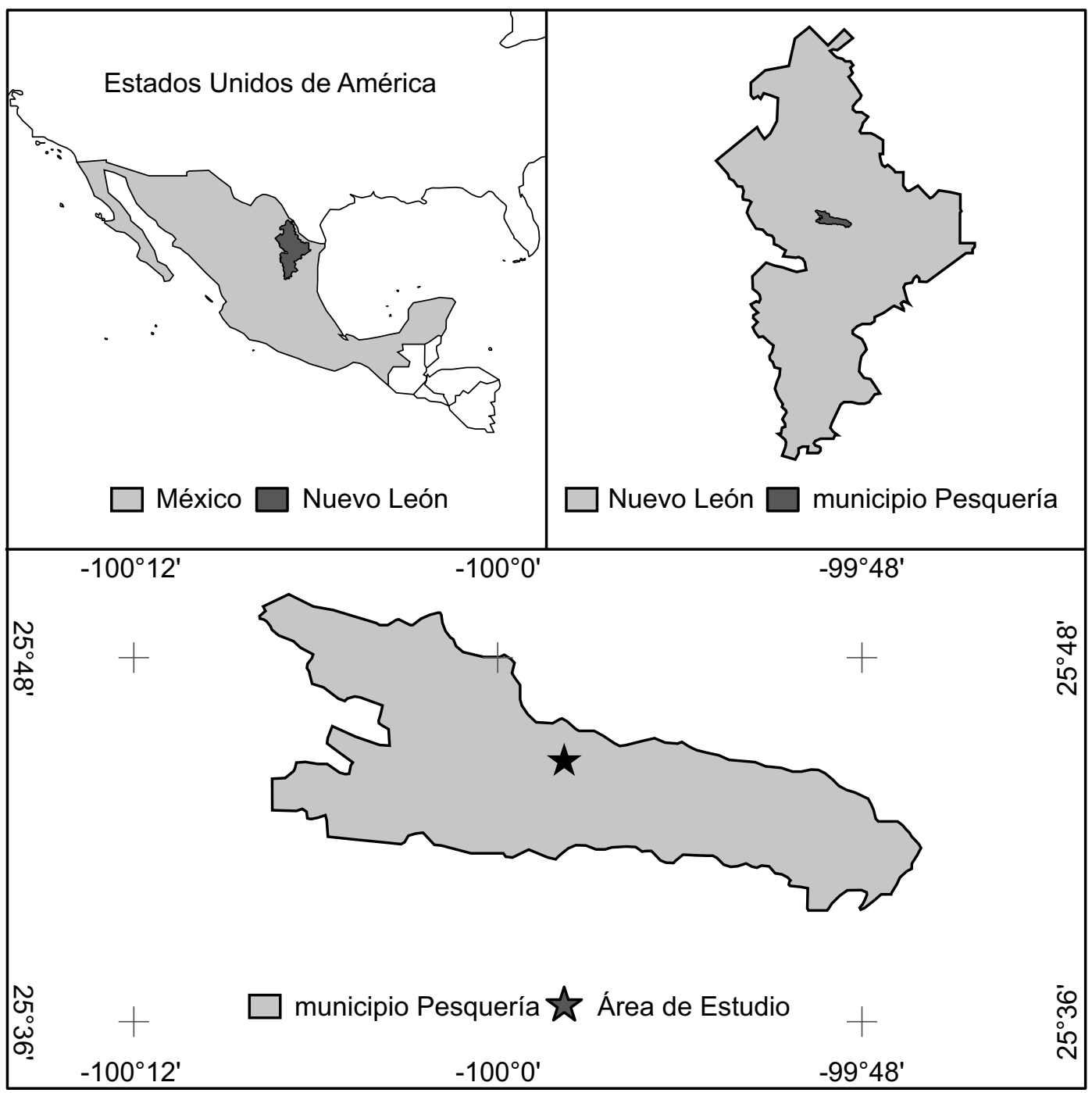

Figura 1: En la imagen superior izquierda se aprecia México, en la derecha el estado de Nuevo León y en la imagen inferior el municipio Pesquería señalando con un punto el área de estudio.

de uso agrícola, donde la vegetación nativa fue eliminada con maquinaria en 1977 y se utilizó para la agricultura de temporal durante 34 años; en el 2013 fue abandonada. En el momento de la evaluación tenía una cobertura de copa de $832 \mathrm{~m} / \mathrm{ha}$. En cada sitio se establecieron cinco cuadrantes de $1 \mathrm{~m}^{2}$ (con un total de 25 cuadrantes para cada condición). En los cuadrantes se midió el diámetro de la cobertura del dosel, en sentido norte-sur y este-oeste para cada individuo de todas las especies herbáceas y arbustivas emergentes, y se realizó la identificación de cada una de éstas. La identificación de las especies se realizó por personal calificado de la Facultad de Ciencias Forestales de la UANL y se revisó la información taxonómica en la Base de Datos Tropicos (TROPICOS, 2017).

\section{Análisis de la vegetación}

De acuerdo con el número de individuos se determinó la densidad (N/ha), con la cobertura se estimó la dominancia $\left(\mathrm{m}^{2} / \mathrm{ha}\right)$ y la frecuencia con base en la presencia en los sitios de muestreo. Los resultados se utilizaron para obtener un valor ponderado a nivel de taxon denominado Índice de Valor de Importancia (IVI), que adquiere valores 
porcentuales en una escala de 0 a 100 (Magurran, 1988). Para evaluar la diversidad se estimó el Índice de Shannon (Shannon, 1948).

Para evaluar cuáles son las principales variables abióticas que están influenciando la presencia y distribución de Amoreuxia wrightii y especies asociadas, se realizó la ordenación de la vegetación (análisis de gradiente directo) mediante el Análisis de Correspondencia Canónica utilizando el programa estadístico CANOCO (ter Braak, 1986).

\section{Variables ambientales edáficas}

En cada una de las dos asociaciones vegetales se establecieron aleatoriamente 25 cuadrantes para evaluar la vegetación. En cada una de estas parcelas se tomaron muestras de suelos de las cuatro esquinas a una profundidad de 20 $\mathrm{cm}$. Se cuantificaron las variables $\mathrm{pH}$, textura, materia orgánica, y densidad aparente, para analizar cuáles variables tienen el mayor impacto sobre la distribución de la vegetación y en especial sobre Amoreuxia wrightii.

\section{Textura}

Se aplicó el método AS-09 NOM-021-RECNAT-2000 (SEMARNAT, 2000), mediante el densímetro de Bouyoucos Gay Lussac (Marca ROBSAN, México). El análisis mecánico es la determinación del porcentaje de arena, limo y arcilla. Estos análisis están basados en la proporción diferencial de asentamiento de las partículas del suelo en el agua.

\section{Materia orgánica}

Se obtuvo a través del método "combustión húmeda y titulación según Walkley/Black modificado", que consiste en la oxidación del carbono orgánico que contiene el suelo con un exceso de dicromato de potasio en un medio fuertemente ácido, valorando el exceso de dicromato con sulfato ferroso (Walkley y Black, 1934).

\section{pH}

La medición de la reacción de pH del suelo se basó en la Norma Oficial Mexicana NOM-021-RECNAT-2000 (SEMARNAT, 2000), por el método AS-23.

\section{Densidad aparente}

Se define como la masa por unidad de volumen (Porta et al., 1999). Indica la compactación del suelo, el grado de facilidad o dificultad que podrían tener las raíces para penetrar el suelo (Castellanos et al., 2000), e inferir las dificultades de emergencia y circulación del agua y el aire (Porta et al., 1999) Se tomaron muestras con cilindro metálico de $4 \times 5 \times 5 \mathrm{~cm}$.

\section{Resultados}

\section{Caracterización del hábitat}

De acuerdo a la relación con otras especies, Amoreuxia wrightii se asoció con especies pioneras que emergen después de un disturbio, con el dominio de especies de herbáceas y pastos como Gutierrezia sarothrae (Pursh) Britton \& Rusby, Gymnosperma glutinosum (Spreng.) Less., Parthenium hysterophorus L., Aristida purpurea Nutt., Panicum obtusum Kunth y Cynodon dactylon (L.) Pers.; así como arbustos de porte bajo como Acacia amentacea, Acacia berlandieri Benth. y Eysenhardtia texana Scheele.

En el área provista de vegetación la especie más abundante resultó ser Panicum obtusum (13.07\%); con mayor cobertura relativa fueron las arbustivas Zanthoxylum fagara (L.) Sarg. (6.02\%), Eysenhardtia texana (5.87\%), Castela texana (Torr. \& A. Gray) Rose var. texana (5.72\%), Karwinskia humboldtiana (Schult.) Zucc. (5.30\%) y Lantana macropoda Torr. (5.24\%); la de mayor frecuencia relativa $(9.09 \%)$ e índice de valor de importancia (7.64\%) fue Gutierrezia sarothrae.

\section{Diversidad}

En los 10 sitios evaluados se registró un total de 26 familias, 44 géneros y 48 especies de plantas vasculares (Apéndice); las cinco familias con mayor número de especies son Leguminosae (9), Asteraceae (5), Poaceae (4), Euphorbiaceae (4) y Verbenaceae (3). Los resultados del Índice de Shannon son de 2.88 para el área no provista de vegetación y de 3.20 para la provista de vegetación. 


\section{Parámetros estructurales}

El Apéndice muestra los resultados de los parámetros estructurales estimados. En los sitios desprovistos de vegetación la especie más abundante y con mayor frecuencia relativa fue Amoreuxia wrightii $(21.07 \% \mathrm{y}$ $14.14 \%$, respectivamente) y Gutierrezia sarothrae tuvo la mayor cobertura relativa $(6.66 \%)$. De acuerdo al Índice de Valor de Importancia (IVI) calculado, Amoreuxia wrightii (12.93\%) resultó ser la especie con el valor más alto.

En el área provista de vegetación la especie más abundante fue Panicum obtusum (13.07\%); con mayor cobertura relativa, Zanthoxylum fagara $(6.02 \%)$ y la de mayor frecuencia relativa $(9.09 \%)$ e índice de valor de importancia (7.64\%), Gutierrezia sarothrae.

\section{Análisis de suelo}

Los suelos de los cinco sitios provistos de vegetación presentaron valores promedios de $\mathrm{pH} 7.44$, una clase textural de franco-arenosa, un valor de contenido de materia orgánica (MO) de 3.15, los cuales se consideran valores muy altos de acuerdo a la tabla de valoración de Walkley y Black Modificado (Cuadro 1). Debido a los aportes constantes de MO por la vegetación del lugar, predominan los colores oscuros con un valor de 5 o menos, siendo el más común el pardo oscuro (10YR 4/2) y una densidad aparente de $0.94 \mathrm{~g} / \mathrm{cm}^{3}$ (Cuadro 2).

Los suelos de áreas sin vegetación presentaron valores promedio de $\mathrm{pH}$ de 7.76 , una clase textural de franco-arcillosa a arcillosa, un contenido de materia orgánica

Cuadro 1: Valoración de contenido de carbono (C) orgánico por tipo de suelo.

\begin{tabular}{lccc}
\hline Contenido de C orgánico & Arenosos & Francos & Arcillosos \\
\hline Muy escaso & $<0.25$ & $<0.50$ & $<0.75$ \\
Escaso & $0.25-0.50$ & $0.50-0.75$ & $0.75-1.00$ \\
Mediano & $0.50-0.75$ & $0.75-1.50$ & $1.00-2.00$ \\
Alto & $0.75-1.50$ & $1.50-2.50$ & $2.00-4.00$ \\
Muy alto & $>1.50$ & $>2.50$ & $>4.00$ \\
\hline
\end{tabular}

bajo (1.28), con colores más claros como el pardo 10YR $5 / 3$ y una densidad aparente de $1.33 \mathrm{~g} / \mathrm{cm}^{3}$ (Cuadro 2). Esto equivale a suelos compactos con poca porosidad en su composición y una infiltración lenta.

\section{Análisis de gradiente directo}

$\mathrm{Al}$ incluir variables bióticas y abióticas en el análisis, mediante CANOCO (Análisis de Correspondencia Canónica, de gradiente directo) se observó que la inercia total de los datos (total inertia $=2.25$ ) es estadísticamente heterogénea y pueden analizarse para obtener resultados referentes a la preferencia de hábitat de las especies analizadas, en especial la especie de estudio Amoreuxia wrightii por determinado tipo de hábitat. Los tres primeros ejes engloban $0.38,0.36$ y 0.25 de la varianza total de los datos y las correlaciones fueron bastante altas, $0.97,0.99$ y 0.96 , lo que demuestra la relación estrecha entre vegetación y variables ambientales (Cuadro 3).

Cuadro 2: Características de los suelos en las áreas desprovistas y provistas de vegetación.

\begin{tabular}{lcc}
\hline Característica & $\begin{array}{c}\text { Desprovisto de } \\
\text { vegetación }\end{array}$ & $\begin{array}{c}\text { Provisto de } \\
\text { vegetación }\end{array}$ \\
\hline $\mathrm{pH}$ & 7.76 & 7.44 \\
Materia orgánica $(\% \mathrm{C})$ & 1.28 & 3.15 \\
Densidad aparente $\mathrm{g} / \mathrm{cm}^{3}$ & 1.33 & 0.94 \\
Clase textural & Franco-Arcillosa & Franco-Arenosa \\
\hline
\end{tabular}

Cuadro 3: Resultados del Análisis de Correspondencia Canónica mostrando los valores de los tres primeros ejes, sus correlaciones y la inercia total (varianza total).

\begin{tabular}{lcccc}
\hline Ejes & 1 & 2 & 3 & $\begin{array}{c}\text { Inercia } \\
\text { total }\end{array}$ \\
\hline Eigenvalores & 0.38 & 0.36 & 0.25 & 2.25 \\
Correlación Espacio-Ambiental & 0.97 & 0.99 & 0.96 & \\
\% varianza acumulada especies & 16.9 & 33.0 & 44.4 & \\
$\begin{array}{l}\text { \% varianza acumulada especies- } \\
\text { ambiente }\end{array}$ & 27.7 & 54.0 & 72.9 & \\
\hline
\end{tabular}


En la figura 2, Amoreuxia wrightii se registró principalmente en los sitios 2,3 y 4 , representando vegetación típica de suelos perturbados, mientras que en los sitios no perturbados 7, 8, 9 y 10 estuvo ausente. En este sentido Amoreuxia se asoció principalmente con Gutierrezia sarothrae, Cynodon dactylon, Carlowrightia texana Henrickson \& T.F. Daniel, Aristida purpurea, Indigofera miniata Ortega, Cenchrus ciliaris L. y Cordia boissieri, especies indicadoras de disturbio (Correll y Johnston, 1970), que habitan en áreas con coberturas aéreas menos densas o sitios con vegetación casi nula.
De acuerdo con las variables evaluadas, se observó que Amoreuxia wrightii prefiere suelos arcillosos y limosos, con suelos con $\mathrm{pH}$ ligeramente alcalinos y con densidad aparente alta. Ningún individuo de Amoreuxia wrightii fue registrado en sitios que presentaron suelos con altos contenidos de arena y altos contenidos de materia orgánica. De igual forma, pero con respecto a la flora asociada en estos sitios, se confirma que Amoreuxia wrightii no está asociada dentro de sitios no perturbados con la vegetación nativa y cobertura aérea densa, especialmente aquella constituida por Jatropha dioica Sessé, Karwinskia humboldtiana, Acacia schaffneri (S. Watson)

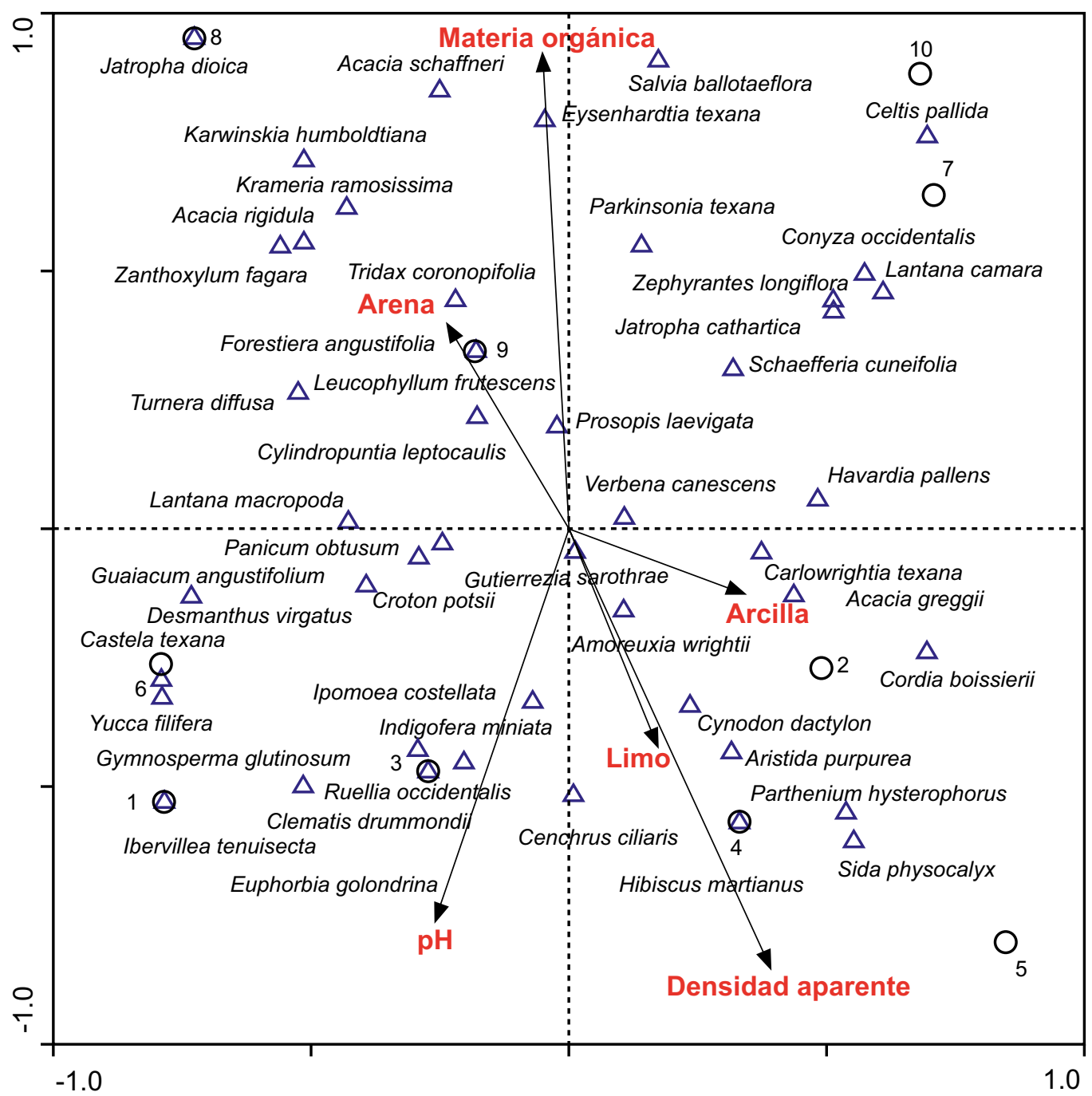

Figura 2: Diagrama (Triplot) de la ordenación entre variables ambientales, sitios y especies de acuerdo con la Ordenación Canónica de Comunidades (CANOCO, por sus siglas en inglés) (ter Braak, 1986). 
F.J. Herm., Acacia amentacea, Zanthoxylum fagara, Leucophyllum frutescens y Forestiera angustifolia Torr.

\section{DISCUSIÓN}

La mayoría de las especies del género Amoreuxia están adaptadas morfológica y fisiológicamente para subsistir en condiciones extremas de sequía en las zonas áridas donde se encuentran (Chávez et al., 2013). Figueroa y Galeano (2007) señalan que una de esas adaptaciones es la presencia de raíces tuberosas que le permiten acumular agua para sobrevivir durante épocas secas. Chávez et al. (2013) menciona que Amoreuxia wrightii tiene un sistema subterráneo complejo, formado por un eje engrosado cuya porción proximal corresponde a un xilopodio de origen posiblemente caulinar y el resto a una estructura tuberosa de origen radical. Además, se presentan raíces laterales con función de reserva, sostén y absorción, lo que asegura la propagación de esta especie en condiciones extremas.

Milanez y Moraes-Dallaqua (2003) y Vilhalva y Apezzato-da-Glória (2006) hacen referencia al desarrollo de un xilopodio de origen caulinar, radical o mixto en estructuras subterráneas en diferentes especies de Asteraceae. Gregory (2006) menciona el desarrollo de reservorios de almidón en las raíces tal como ocurre en Amoreuxia wrightii, las cuales proporcionan alimento a la planta durante periodos de sequía.

Amoreuxia wrightii se asoció principalmente con Gutierrezia sarothrae, Cynodon dactylon, Carlowrightia texana, Aristida purpurea, Indigofera miniata, Cenchrus ciliaris y Cordia boissieri, todas indicadoras de disturbio (Correll y Johnston, 1970) y que habitan en áreas con coberturas aéreas menos densas o sitios sin vegetación. También se asoció a arbustos de porte bajo como Acacia amentacea, Acacia berlandieri y Eysenhardtia texana. Estos arbustos son los primeros en regenerar después de actividades productivas o perturbación (Alanís et al., 2008; Alanís et al., 2013; Jiménez et al., 2013).

El suelo del área con escasa vegetación, que fue donde se encontró $A$. wrightii, muestra valores ligeramente alcalinos, no salinos y arcillosos. Cutler (1977) y Dickison (2000) reportaron suelos muy similares en donde crece Amoreuxia wrightii, señalando que, en ambientes áridos con suelos arcillosos, con escasa precipitación y alta evapotranspiración, es frecuente que presenten estructuras subterráneas capaces de almacenar sustancias de reserva en algunos casos o agua en otros.

Lo anterior se ve reflejado en el índice de valor de importancia, ya que para la zona desprovista de vegetación, en donde los suelos son arcillosos especies como Amoreuxia wrightii presentan el valor más altos de densidad. Caso contrario en el área provista de vegetación, la especie mostró valores menores. Además del tipo de suelo, $A$. wrightii se estableció con mayor abundancia en el área no provista de vegetación, condiciones en las que de acuerdo con Raposo et al. (2016) existe una escasa cobertura vegetal y condición para que se desarrollen las especies heliófitas.

Los estudios sistemáticos sobre la distribución, ecología, germinación y propagación de Amoreuxia son escasos. Por lo anterior se considera necesario desarrollar más investigaciones sobre estos temas. A nivel especie, se han generado, en Venezuela, estudios de Amoreuxia wrightii que evalúan aspectos biológicos y poblacionales (Chávez et al., 2016) y anatomía foliar y del sistema subterráneo (Chávez et al., 2013), pero hace falta generar conocimiento de su germinación y propagación para fines de preservación y producción de especies.

\section{CONCLUSIONES}

Amoreuxia wrightii fue más abundante en áreas con nula cobertura, desprovistas de vegetación o bien, áreas abiertas, sobre suelos con bajo contenido de materia orgánica y altos contenidos de $\mathrm{pH}$, limo, arcilla y densidad aparente. De acuerdo a la relación con otras especies, se asoció con especies pioneras, que emergen después de un disturbio, con el dominio de especies de herbáceas y pastos. En las áreas sin perturbación, con suelos donde hay contenidos altos de materia orgánica y que albergan vegetación arbustiva con cobertura aérea densa, se encontró una población menor de Amoreuxia wrightii. 


\section{CONTRIBUCIONES DE AUTORES}

BSM, EAR, EJ y MPM concibieron y diseñaron el estudio. BSM y EAR realizaron los análisis. BSM, VMG, AAR y JJMC contribuyeron a la adquisición de datos y la interpretación. BSM y EAR escribieron el manuscrito con la ayuda de EJ, MPM, VMG, AAR y JJMC. Todos los autores contribuyeron a la discusión, revisión y aprobación del manuscrito final.

\section{FINANCIAMIENTO}

Se agradece al Consejo Nacional de Ciencia y Tecnología por haberle otorgado una beca de manutención a la primera autora. También se agradece el apoyo de la empresa RENAC, S.A. de C.V. por haber apoyado con la logística de las actividades en campo, haber cubierto la adquisición de los reactivos de laboratorio y los gastos de transporte y alimentación de las brigadas de campo.

\section{LITERATURA CITADA}

Alanís, E., J. Jiménez, O. A. Aguirre, J. E. Treviño, E. Jurado y M. A. González. 2008. Efecto del uso del suelo en la fitodiversidad del matorral espinoso tamaulipeco. Ciencia UANL 11(1): 56-62.

Alanís, E., J. Jiménez, M. A. González, J. I. Yerena, L. G. Cuellar y A. Mora-Olivo. 2013. Análisis de la vegetación secundaria del matorral espinoso tamaulipeco, México. Phyton, Revista Internacional de Botánica Experimental 82(2): 185-191.

Calderón de Rzedowski, G. 1994. Cochlospermaceae. Flora del Bajío y de regiones adyacentes 95: 1-5.

Castellanos J. Z., J. X. Uvalle y A. Aguilar. 2000. Manual de interpretación de análisis de suelos y aguas. 2a. Ed. Instituto de Capacitación para la Productividad Agrícola. San Miguel de Allende, México. 226 pp.

Cedano Maldonado, M. 2000. Revisión de la familia Cochlospermaceae para México. Tesis de maestría en Ciencias Biológicas. Área de sistemática vegetal, Universidad de Guadalajara. Guadalajara, México. 241 pp.

Chávez J., D. Jáuregui, M. Lapp y P. Torrecilla. 2013. Anatomía foliar y del sistema subterráneo de Amoreuxia wrightii
A. Gray (Bixaceace), especies en peligro crítico en Venezuela. Ernstia 23(1): 47-65.

Chávez, J., M. Lapp, P. Torrecilla, R. Wingfield y L. García. 2016. Aspectos biológicos y poblacionales con relación al estado de amenaza local de Amoreuxia wrightii A. Gray (Bixaceae), especie de distribución restringida en Venezuela. Ernstia 26(1): 39-73.

Correll, D. S. y M. C. Johnston. 1970. Manual of the vascular plants of Texas. Texas research foundation. Renner, USA. Pp. 36-84.

Cutler, D. F. 1977. Applied plant anatomy. Longman. London and New York, UK, USA. 103 pp.

Dickison, W. C. 2000. Integrative plant anatomy. Academic Press. San Diego, USA. 533 pp.

Estrada, A. E., C. Yen, A. Delgado y J. A. Quintanilla. 2004. Leguminosas del centro del estado de Nuevo León, México. Universidad Nacional Autónoma de México, Anales del Instituto de Biología, Serie Botánica 75: 73-85. Figueroa, Y. y G. Galeano. 2007. Lista comentada de las plantas vasculares del Enclave Seco Interandino de la Tatacoa (Huila, Colombia). Caldasia 29(2): 263-281.

Gregory, P. J. 2006. Plant Roots: Growth, activity and interaction with soils. Blackwell Publishing. Oxford, UK. 318 pp. DOI: http://dx.doi.org/10.1002/9780470995563.ch1

Henrickson, J. y M. C. Johnston. 1997 (inédito). A flora of the Chihuahuan Desert region. Los Angeles, USA. 1687 pp.

INEGI. 1986. Síntesis Geográfica de Nuevo León. Secretaría de Programación y Presupuesto. México, D.F., México. $170 \mathrm{pp}$.

INEGI. 2014. Perspectiva estadística Nuevo León. Instituto Nacional de Estadística y Geografía. México, D.F., México. 95 pp.

Jiménez, J., E. Alanís, M. A. González, O. A. Aguirre, E. J. Treviño. 2013. Characterizing regeneration of woody species in areas with different land-history tenure in the Tamaulipan thornscrub, Mexico. The Southwestern Naturalist 58(3): 299-304. DOI: http://dx.doi. org/10.1894/0038-4909-58.3.299

Magurran, A. E. 1988. Ecological Diversity and Its Measurements. Chapman \& Hall. London, UK. 179 pp. DOI: http://dx.doi.org/10.1007/978-94-015-7358-0 
Milanez, C. y M. Moraes-Dallaqua. 2003. Ontogênese do sistema subterráneo de Pachyrhizus ahipa (Wedd.) Parodi (Fabaceae). Revista Brasileira de Botânica 26(3): 415-427. DOI: http://dx.doi.org/10.1590/S010084042003000300014.

Pío-León, J. F., J. L. León-de la Luz y A. Ortega-Rubio 2014. Nuevo registro de Amoreuxia gonzalezii (Bixaceae) para la península de Baja California, México. Revista Mexicana de Biodiversidad 85(4): 1269-1272. DOI: http://dx.doi. org/10.7550/rmb.44212

Poppendieck, H. H. 1980. A monograph of the Cochlospermaceae. Botanische Jahrbuecher fuer Systematik Pflanzengeschichte und Pflanzengeographie 101(2): 191-265.

Poppendieck, H. H. 1981. Cochlospermaceae. Flora Neotropical Monograph 27: 1-34.

Porta, J., M. López-Acevedo y C. Roquero. 1999. Edafología para la agricultura y el medio ambiente. 2a. ed. Ed. Mundi-Prensa. Asturias, España. Pp. 155, 256.

Raposo, M., P. Mendes, A. Cano-Ortiz y C. Pinto-Gomes. 2016. Séries de vegetação prioritárias para a conservação no centro e sul de Portugal continental. Botanique 1: 113148.

SEMARNAT. 2000. Norma Oficial Mexicana NOM-021RECNAT-2000. Que establece las especificaciones de fertilidad, salinidad y clasificación de suelos. Estudios, muestreo y análisis. Secretaría de Medio Ambiente y Recursos Naturales. Diario Oficial de la Federación. Cd. Mx., México.

SEMARNAT. 2010. Norma Oficial Mexicana NOM 059-SEMARNAT-2010. Protección ambiental-Especies nativas de México de flora y fauna silvestres-Categorías de riesgo y especificaciones para su inclusión, exclusión o cambio-Lista de especies en riesgo. Secretaría de Medio Ambiente y Recursos Naturales. Diario Oficial de la Federación. Cd. Mx., México. http://dof.gob.mx/nota_ detalle.php?cod igo $=5173091 \&$ fecha $=30 / 12 / 2010$

Shannon, C. E. 1948. A mathematical theory of communication. The Bell System Technical Journal 27: 379-423, 623-656. Sousa Pereira, D., M. Sousa Pereira y A. M. Esmeraldo Bezerra. 2013. Effect of light and temperature on seed germination of Cochlospermum vitifolium (Will.) Sprengel. Floresta e Ambiente 20(3): 391-397. DOI: http://dx.doi.org/10.4322/ floram.2013.026

ter Braak, C. J. F. 1986. Canonical Correspondence Analysis: A new eigenvector technique for multivariate direct gradient analysis. Ecology 67(5): 1167-1179. DOI: http://dx.doi. org/10.2307/1938672

TROPICOS. 2017. Tropicos.org. Missouri Botanical Garden. http://ww.tropicos.org

Velazco, C. G. 2009. Flora del estado de Nuevo León, México: Diversidad y Análisis Espacio-Temporal. Tesis doctoral. Facultad de Ciencias Biológicas, Universidad Autónoma de Nuevo León. San Nicolás de Los Garza, México. 218 pp.

Vilhalva, D. y B. Apezzato-da-Glória. 2006. Morfo-anatomia do sistema subterrâneo de Calea verticillata (Klatt) Pruski e Isostigma megapotamicum (Spreng.) Sherff-Asteraceae. Revista Brasileira de Botânica 29(1): 39-47. DOI: http:// dx.doi.org/10.1590/S0100-84042006000100005

Walkley, A. e I. A. Black. 1934. An examination of the Degtajareff method for determining soil organic matter and a proposed modification of the chromic acid titration method. Soil Science 37(1): 29-38. DOI: http://dx.doi. org/10.1097/00010694-193401000-00003 
Apéndice. Abundancia, dominancia, frecuencia e índice de valor de importancia de las especies registradas para cada condición.

\begin{tabular}{|c|c|c|c|c|c|c|c|c|c|}
\hline \multirow{2}{*}{ Familia } & \multirow[b]{2}{*}{ Especies } & \multicolumn{4}{|c|}{ No provistas de vegetación } & \multicolumn{4}{|c|}{ Provistas de vegetación } \\
\hline & & 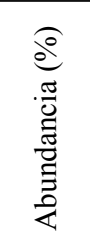 & 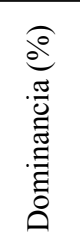 & 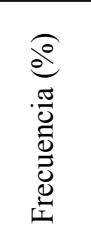 & $\sum$ & 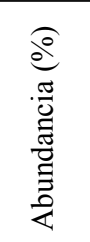 & 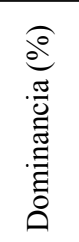 & 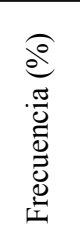 & $\sum$ \\
\hline \multirow[t]{2}{*}{ ACANTHACEAE } & $\begin{array}{l}\text { Carlowrightia texana Henrickson \& T.F. } \\
\text { Daniel }\end{array}$ & 6.13 & 3.00 & 1.53 & 3.55 & 0.00 & 0.00 & 0.00 & 0.00 \\
\hline & $\begin{array}{l}\text { Ruellia occidentalis (A. Gray) Tharp \& } \\
\text { F.A. Barkley }\end{array}$ & 0.38 & 0.26 & 0.76 & 0.47 & 0.00 & 0.00 & 0.00 & 0.00 \\
\hline AGAVACEAE & Yucca filifera Chabaud & 0.38 & 1.76 & 0.76 & 0.97 & 0.65 & 2.26 & 1.01 & 1.31 \\
\hline AMARYLLIDACEAE & Zephyranthes longifolia Hemsl. & 2.68 & 2.12 & 2.3 & 2.37 & 5.88 & 1.50 & 2.02 & 3.14 \\
\hline \multirow[t]{5}{*}{ ASTERACEAE } & Conyza canadensis (L.) Cronquist & 0.38 & 1.13 & 0.76 & 0.76 & 1.96 & 2.60 & 2.02 & 2.19 \\
\hline & $\begin{array}{l}\text { Gutierrezia sarothrae (Pursh) Britton \& } \\
\text { Rusby }\end{array}$ & 10.73 & 6.66 & 10.76 & 9.38 & 10.46 & 3.37 & 9.09 & 7.64 \\
\hline & Gymnosperma glutinosum (Spreng.) Less. & 3.45 & 2.01 & 4.61 & 3.36 & 0.00 & 0.00 & 0.00 & 0.00 \\
\hline & Parthenium hysterophorus L. & 6.51 & 1.70 & 4.61 & 4.27 & 1.31 & 3.31 & 1.01 & 1.88 \\
\hline & Tridax coronopifolia (Kunth) Hemsl. & 0.77 & 1.07 & 0.76 & 0.87 & 0.65 & 1.56 & 1.01 & 1.08 \\
\hline BORAGINACEAE & Cordia boissieri A. DC. & 0.77 & 1.05 & 1.53 & 1.11 & 0.65 & 4.51 & 1.01 & 2.06 \\
\hline BIXACEAE & Amoreuxia wrightii A. Gray & 21.07 & 3.57 & 14.14 & 12.93 & 11.11 & 1.28 & 6.06 & 6.15 \\
\hline CACTACEAE & $\begin{array}{l}\text { Cylindropuntia leptocaulis (DC.) F.M. } \\
\text { Knuth }\end{array}$ & 0.00 & 0.00 & 0.00 & 0.00 & 1.31 & 1.70 & 2.02 & 1.68 \\
\hline CELASTRACEAE & Schaefferia cuneifolia A. Gray & 0.77 & 1.07 & 1.53 & 1.12 & 1.31 & 2.14 & 2.02 & 1.82 \\
\hline CUCURBITACEAE & Ibervillea tenuisecta (A. Gray) Small & 0.38 & 3.78 & 0.76 & 1.64 & 0.00 & 0.00 & 0.00 & 0.00 \\
\hline CONVOLVULACEAE & Ipomoea costellata Torr. & 1.15 & 4.85 & 2.3 & 2.77 & 0.00 & 0.00 & 0.00 & 0.00 \\
\hline \multirow[t]{4}{*}{ EUPHORBIACEAE } & Croton pottsii (Klotzsch) Müll. Arg. & 3.83 & 1.27 & 3.48 & 2.86 & 5.88 & 0.71 & 6.06 & 4.22 \\
\hline & Euphorbia golondrina L.C. Wheeler & 0.38 & 3.13 & 0.76 & 1.43 & 0.00 & 0.00 & 0.00 & 0.00 \\
\hline & Jatropha cathartica Terán \& Berland. & 0.38 & 0.77 & 0.76 & 0.64 & 1.96 & 3.39 & 3.03 & 2.79 \\
\hline & Jatropha dioica Sessé & 0.00 & 0.00 & 0.00 & 0.00 & 3.92 & 2.32 & 1.01 & 2.42 \\
\hline KRAMERIACEAE & $\begin{array}{l}\text { Krameria ramosissima (A. Gray) S. } \\
\text { Watson }\end{array}$ & 0.00 & 0.00 & 0.00 & 0.00 & 1.96 & 4.48 & 2.02 & 2.82 \\
\hline LAMIACEAE & Salvia ballotiflora Benth. & 0.00 & 0.00 & 0.00 & 0.00 & 2.61 & 1.05 & 3.03 & 2.23 \\
\hline \multirow[t]{8}{*}{ LEGUMINOSAE } & Acacia greggii A. Gray & 0.38 & 5.36 & 0.76 & 2.17 & 0.65 & 2.41 & 1.01 & 1.36 \\
\hline & Acacia rigidula Benth. & 0.38 & 0.34 & 0.76 & 0.50 & 2.61 & 1.63 & 4.04 & 2.76 \\
\hline & Acacia schaffneri (S. Watson) F.J. Herm. & 0.00 & 0.00 & 0.00 & 0.00 & 1.96 & 0.78 & 3.03 & 1.92 \\
\hline & Desmanthus virgatus (L.) Willd. & 2.30 & 6.48 & 3.07 & 3.95 & 2.61 & 3.91 & 2.02 & 2.85 \\
\hline & Eysenhardtia texana Scheele & 0.00 & 0.00 & 0.00 & 0.00 & 1.96 & 5.87 & 3.03 & 3.62 \\
\hline & Havardia pallens (Benth.) Britton \& Rose & 0.38 & 1.07 & 0.76 & 0.74 & 0.65 & 3.39 & 1.01 & 1.68 \\
\hline & Indigofera miniata Ortega & 3.07 & 5.11 & 2.3 & 3.49 & 1.96 & 1.24 & 1.01 & 1.40 \\
\hline & Parkinsonia texana (A. Gray) S. Watson & 0.38 & 7.30 & 0.76 & 2.81 & 1.31 & 0.97 & 2.02 & 1.43 \\
\hline
\end{tabular}


Apéndice. Continuación.

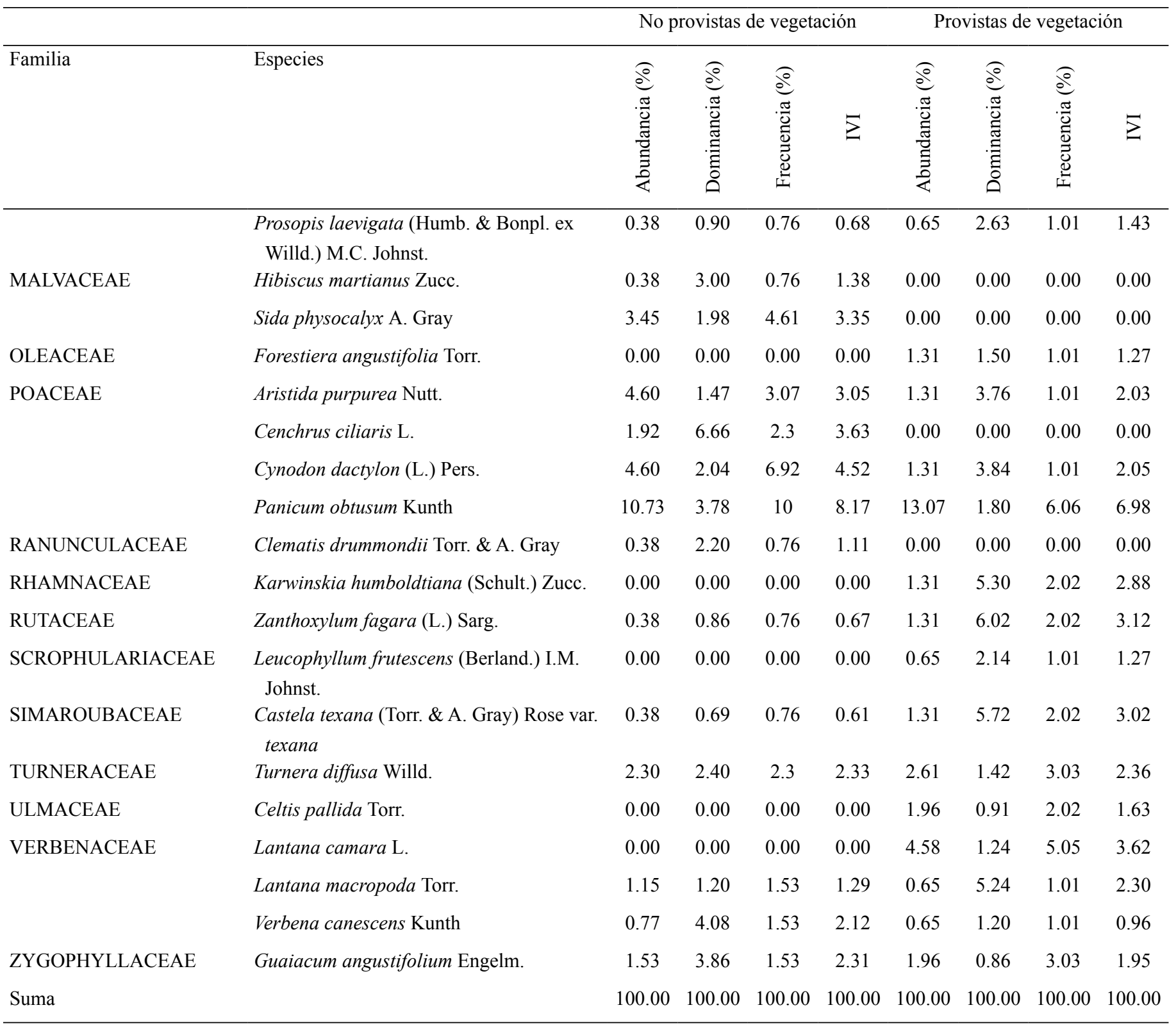

